\title{
Emotion regulation during pregnancy: a call to action for increased research, screening, and intervention
}

\author{
Francesca Penner $^{1}$ (D) Helena J. V. Rutherford ${ }^{1}$
}

Received: 29 September 2021 / Accepted: 5 January 2022 / Published online: 11 January 2022

(c) The Author(s), under exclusive licence to Springer-Verlag GmbH Austria, part of Springer Nature 2022

\begin{abstract}
This paper serves as a call to action for increased focus on emotion regulation during pregnancy. We make this case by summarizing the limited research to date on this topic, which has demonstrated that emotion regulation in pregnant people has important mental health, caregiving, and developmental correlates throughout the perinatal period. Given its crosscutting and modifiable nature, bolstering emotion regulation during pregnancy has the potential for considerable intergenerational consequences, and it is critical to further investigate this construct.
\end{abstract}

Keywords Emotion regulation · Pregnancy $\cdot$ Perinatal mental health $\cdot$ Interventions $\cdot$ Prenatal period $\cdot$ Expectant parents

Pregnancy is a time of significant neurobiological and psychological change, often associated with stress and increased risk for psychopathology. Given the salience of stress and mental health during this period, there is a burgeoning interest in emotion regulation during pregnancy. While multiple, but related, definitions of emotion regulation exist, here we refer to emotion regulation (ER) as concerning one's attempts to influence, modulate, or manage the emotions one is experiencing and how they are expressed (Gross and Muñoz 1995, p. 152). In particular, our lens on ER is focused primarily on behaviors or ER strategies-which may be adaptive or maladaptive-used to regulate emotions, as these have been shown to be a core factor across domains of psychopathology and are targets of change across multiple mental health interventions (Sloan et al. 2017). ER strategies may be focused on the antecedent (e.g., event, stimulus, or stressor that evokes the emotion) or on one's emotional response (Gross and Muñoz 1995). In the context of pregnancy, considering that pregnant people may experience a number of stressors and physiological and emotional changes, the ability to effectively use ER strategies to manage negative emotional states may help to prevent or reduce distress and psychopathology during this period, and may

Francesca Penner

Francesca.Penner@yale.edu

1 Yale Child Study Center, 230 S. Frontage Rd, New Haven, CT 06520, USA also have implications for postpartum parental health, caregiving, and child health.

At the time of our writing, the COVID-19 pandemic continues to place additional stressors on parents and elevate physical and mental health risks among pregnant people. Therefore, it is more important than ever to understand and bolster ER during pregnancy. The current paper serves as a call to action for increased focus on ER during pregnancy in both research and healthcare settings. We support this argument by summarizing early evidence on correlates of prenatal ER, and highlighting existing evidence-based interventions that may promote ER during pregnancy. We conclude with gaps and future directions to lay the groundwork for future research in this area.

\section{Measurement of emotion regulation during pregnancy}

Studies that have measured ER during pregnancy have primarily used self-report measures applicable to adults in general, namely, the Difficulties in Emotion Regulation Scale (e.g., Ostlund et al. 2019), which has been used to measure self-reported problems regulating emotions, and the Emotion Regulation Questionnaire (e.g., Coo et al. 2020), which measures employment of ER strategies of reappraisal (antecedent-focused) and suppression (response-focused). Assessment of respiratory sinus arrythmia (RSA) has also been used as a biological marker of ER during pregnancy 
(Lin et al. 2019). Given that ER skills may change across development, particularly during major developmental windows such as pregnancy and postpartum when physiological changes are occurring (Rutherford et al. 2015), additional research is needed to determine whether perinatal-specific measures of ER are warranted.

\section{Health and mental health of the pregnant person}

Evidence is accumulating for the role of ER during pregnancy in the physical and mental health of pregnant people. Poor ER has been associated with increased hair cortisol in pregnant women (Conradt et al. 2020), as well as with smoking and other substance use (Bradizza et al. 2017), eating behaviors and obesity (de Campora et al. 2014), and sleep problems (Kalmbach et al. 2020) during pregnancy. For individuals experiencing pregnancy-related anxiety (Basharpoor and Taherifard, 2019), high levels of stress (Lin et al. 2019), or worries due to medical complications during pregnancy (McCoyd et al. 2020), ER is also implicated, in that challenges effectively regulating these emotional states during pregnancy may result in their maintenance or exacerbation. Moreover, poor ER prenatally has been associated with higher levels of depression, anxiety, borderline personality pathology, and self-injurious thoughts and behaviors in pregnant women (Lin et al. 2019; Kalmbach et al. 2020).

Prenatal ER may also have downstream effects on a host of postnatal parental-child outcomes through its associations with prenatal health. For example, studies have begun to show longitudinal associations between poor ER during pregnancy and postpartum depression and anxiety (CoO et al. 2020). In general, however, there is a dearth of work examining the effects of prenatal ER on postnatal outcomes.

\section{Child health and development}

There is also growing evidence that ER during pregnancy may have long-term implications for child health and development. For example, researchers have begun to unpack the intergenerational transmission of emotion dysregulation, finding that ER problems in pregnancy are associated with neurobehavioral risks for emotion dysregulation in infants-specifically, lowered arousal and attention (Ostlund et al. 2019). ER during pregnancy has also been linked to the quality of infant feeding interactions (de Campora et al. 2014), suggesting that prenatal ER may impact infant physical health outcomes. Related evidence also suggests that exposure to stress or psychological distress in the pregnant parent can have fetal programming effects and negatively affect multiple aspects of child health and development, including fetal weight, growth, and birth outcomes, child cardiometabolic outcomes, child respiratory and atopic outcomes, and child neurodevelopmental, academic, and mental health outcomes (Vehmeijer et al. 2019). Consequently, promoting ER during pregnancy as a means of reducing distress has the potential to benefit both the pregnant parent and child.

\section{Parenting}

ER during pregnancy has additional intergenerational relevance due to its effects on parenting and the parent-child relationship. ER skills are necessary for many parenting tasks (e.g., sensitively responding to a crying infant; Rutherford et al. 2015), and self-reported problems in ER have been associated with blunted physiological responsivity to an infant cry task during pregnancy (Lin et al. 2019), suggesting that prenatal ER has direct implications for parenting behavior. To the extent that ER is stable from pregnancy to postpartum, prenatal ER may be directly associated with postnatal ER and caregiving behaviors in parents. Research has not yet tested this question.

In terms of parent-child relational effects, ER during pregnancy may be relevant to prenatal attachment, which has been shown to predict postnatal attachment and caregiving quality (Paley and Hajal 2021). When considering ER and the parent-infant relationship, it is important to note that parents' ER postpartum is influenced by the infant and the experience of caregiving (Rutherford et al. 2015), suggesting bidirectional parent-infant effects. More research is needed to elucidate these bidirectional effects postpartum; nevertheless, prenatal interventions may be especially beneficial so that parents can strengthen ER skills before infant effects are introduced (Paley and Hajal 2021).

\section{Interventions to improve emotion regulation during pregnancy}

Pregnancy represents a critical window when improving ER may have the potential to impact multiple parent, infant, and caregiving outcomes. Furthermore, pregnancy itself represents a unique opportunity for intervention when pregnant people are making regular healthcare visits, and before the additional stress of having a newborn is present (Paley and Hajal 2021). Indeed, ER is already a therapeutic target in several interventions delivered during pregnancy, for specific populations of pregnant individuals, including those who smoke, use substances, overeat, have sleep problems, or are in mental health treatment (see Bradizza et al. 2017; Wilson and Donachie 2018). These interventions provide critical evidence that ER can be improved during pregnancy. 
In particular, we highlight three types of evidence-based interventions that consider ER and have been adapted for use with pregnant populations: mindfulness interventions, mentalization-based treatment (MBT), and dialectical behavior therapy (DBT).

Mindfulness interventions are thought to improve ER by increasing mindfulness - or the ability to fully focus on and experience the present moment-in order to reduce emotional reactivity, improve coping, and reduce symptoms of depression and anxiety. Two studies have shown significant improvements in ER following mindfulness intervention in pregnant women (for a review, see Lucena et al. 2020). A MBT approach that begins during pregnancy is Minding the Baby, a program that has been shown to increase parental mentalization, the ability to recognize mental states in one's self and one's child and how this relates to behavior. Mentalization is thought to improve ER by helping parents be more reflective and slow down automatic thoughts, emotions, and behaviors (Slade et al. 2020). DBT has a primary focus on helping individuals manage emotion dysregulation and teaches a repertoire of specific behavioral skills to build emotion regulation abilities; DBT has also been implemented with pregnant and postpartum women, evidencing significant changes in the ability to express and manage emotions (Wilson and Donachie 2018).

While these prenatal interventions have more frequently been delivered to those with specific health symptoms or other pregnancy risks, there may be benefit to universallevel efforts to improve ER in pregnant populations, not only for health promotion but also for early parenting intervention. Such interventions should include psychoeducation on the importance of ER for caregiving and teach and practice ER skills generally and in caregiving-specific scenarios.
Prenatal interventions to promote ER may also benefit from involvement of partners or secondary caregivers to address individual and co-regulation, including discussion of how expectant parents' own attachment relationships may have shaped their emotional functioning (Paley and Hajal 2021). Complementing interventions, it may be fruitful to screen for ER problems among expectant parents, as a means of assessing for potential mental health and caregiving risks, though the utility of this approach remains to be tested. However, the benefit of prenatal ER screening is that expectant parents experiencing emotional distress may be more likely to endorse ER problems compared to symptoms of depression and anxiety, due to stigma associated with these conditions, particularly during pregnancy.

\section{Conclusion and future directions}

Research examining ER during pregnancy is only in the beginning stages but has started to identify important correlates and potential pathways (Fig. 1). While the current paper does not represent a comprehensive review of all studies that have measured ER during pregnancy, we provide a summary of what is known thus far to encourage more research on this topic. We also acknowledge that prenatal ER has conceptual crossover with existing literatures that have larger evidence bases, including research focused on pregnancy-related anxiety, prenatal stress, perinatal depression and anxiety, and medical complications during pregnancy. These crossovers underline not only the relevance of prenatal ER but also the need for more research-particularly given the relative dearth of studies that have formally measured prenatal ER.
Fig. 1 Conceptual model showing correlates of emotion regulation (ER) in pregnancy. Dashed boxes represent variables for which we lack research, though there is a need for more studies investigating all of the correlates shown here. Note that there are likely to be bidirectional associations between prenatal ER and other prenatal variables. It is also likely that prenatal ER indirectly influences postnatal outcomes via its associations with prenatal health and caregiving, in addition to direct effects depicted here

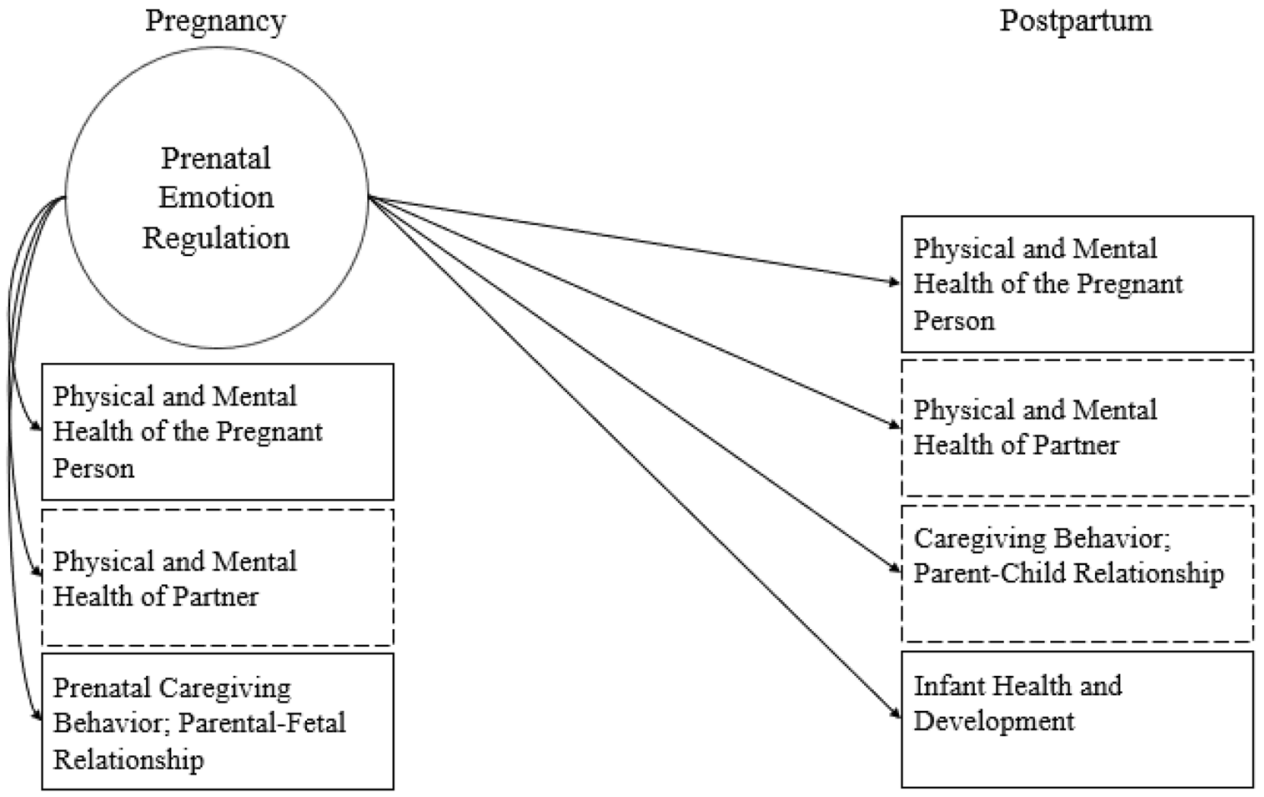


Our summary illustrates that the majority of studies that have empirically evaluated prenatal ER involve crosssectional research conducted during pregnancy. There is a need for research examining how ER during pregnancy affects outcomes postnatally for both the pregnant person and child. Longitudinal research can also help identify how ER may or may not change from pregnancy to postpartum, when bidirectional effects between parent and infant are at play. There is also a need for studies investigating the interplay of ER between pregnant people and their partners.

Research would further benefit from assessing additional biological measures of ER, such as the measurement of event-related potentials, skin conductance, heart rate, or blood pressure during emotion-eliciting or emotion regulation tasks, as a means of understanding ER in pregnant individuals at multiple levels of analysis. Psychometric studies of existing ER questionnaires are also essential to test whether there is measurement invariance for pregnant people compared to other adults. Measurement noninvariance would signify that the emotion regulation construct being measured differs during pregnancy, perhaps suggesting a need for pregnancy- or perinatal-specific ER measures. This work is particularly important considering that brief and valid measurement of ER during pregnancy would better facilitate increased attention to and assessment of this construct in research and prenatal care.

Future research should evaluate the clinical utility of screening for prenatal ER in terms of its ability to predict later mental health or caregiving problems and should continue to test the effectiveness of existing interventions for bolstering ER in pregnant people, particularly when applied universally and/or integrated into obstetric care. Bolstering ER has unique utility within prenatal interventions because it is transdiagnostic, lending itself to universal interventions to impact a variety of concurrent and future parental, child, and family mental and physical health outcomes. Though more research is needed, screening for and promoting ER during pregnancy have the potential for multi-generational impacts.

Funding Dr. Penner was supported by T32 MH018268.

Data availability Not applicable.

Code availability Not applicable.

\section{Declarations}

Conflict of interest The authors declare no competing interests.

\section{References}

Basharpoor S, Taherifard M (2019) Predicting the pregnancy anxiety in pregnant women basing on cognitive biases and emotion regulation strategies. Iran J Psychiatr Nurs 7:23-31

Bradizza CM, Stasiewicz PR, Zhuo Y et al (2017) Smoking cessation for pregnant smokers: development and pilot test of an emotion regulation treatment supplement to standard smoking cessation for negative affect smokers. Nicotine Tob Res off J Soc Res Nicotine Tob 19:578-584. https://doi.org/10.1093/ntr/ntw398

Conradt E, Shakiba N, Ostlund B et al (2020) Prenatal maternal hair cortisol concentrations are related to maternal prenatal emotion dysregulation but not neurodevelopmental or birth outcomes. Dev Psychobiol 62:758-767. https://doi.org/10.1002/dev.21952

Coo S, García MI, Prieto F, Medina F (2020) The role of interpersonal emotional regulation on maternal mental health. J Reprod Infant Psychol 0:1-19. https://doi.org/10.1080/02646838.2020. 1825657

de Campora G, Giromini L, Larciprete G et al (2014) The impact of maternal overweight and emotion regulation on early eating behaviors. Eat Behav 15:403-409. https://doi.org/10.1016/j. eatbeh.2014.04.013

Gross JJ, Muñoz RF (1995) Emotion regulation and mental health. Clin Psychol Sci Pract 2:151-164. https://doi.org/10.1111/j. 1468-2850.1995.tb00036.x

Kalmbach DA, Cheng P, Ong JC et al (2020) Depression and suicidal ideation in pregnancy: exploring relationships with insomnia, short sleep, and nocturnal rumination. Sleep Med 65:62-73. https://doi.org/10.1016/j.sleep.2019.07.010

Lin B, Kaliush PR, Conradt E et al (2019) Intergenerational transmission of emotion dysregulation: Part I. Psychopathology, self-injury, and parasympathetic responsivity among pregnant women. Dev Psychopathol 31:817-831. https://doi.org/10.1017/ S0954579419000336

Lucena L, Frange C, Pinto ACA et al (2020) Mindfulness interventions during pregnancy: a narrative review. J Integr Med 18:470-477. https://doi.org/10.1016/j.joim.2020.07.007

McCoyd JLM, Curran L, Munch S (2020) They say, "If you don't relax...you're going to make something bad happen": women's emotion management during medically high-risk pregnancy. Psychol Women Q 44:117-129. https://doi.org/10.1177/03616 84319883199

Ostlund BD, Vlisides-Henry RD, Crowell SE et al (2019) Intergenerational transmission of emotion dysregulation: Part II. Developmental origins of newborn neurobehavior. Dev Psychopathol 31:833-846. https://doi.org/10.1017/S0954579419000440

Paley B, Hajal NJ (2021) Continuity in early caregiving experiences and the transition to parenthood: role of emotion regulation and coregulation as family-level processes. In: Kuersten-Hogan R, McHale JP (eds) Prenatal family dynamics: couple and coparenting relationships during and postpregnancy. Springer International Publishing, Cham, pp 321-342

Rutherford HJV, Wallace NS, Laurent HK, Mayes LC (2015) Emotion regulation in parenthood. Dev Rev 36:1-14. https://doi.org/ 10.1016/j.dr.2014.12.008

Slade A, Holland ML, Ordway MR et al (2020) Minding the Baby®: enhancing parental reflective functioning and infant attachment in an attachment-based, interdisciplinary home visiting program. Dev Psychopathol 32:123-137. https://doi.org/10.1017/ S0954579418001463

Sloan E, Hall K, Moulding R et al (2017) Emotion regulation as a transdiagnostic treatment construct across anxiety, depression, 
substance, eating and borderline personality disorders: a systematic review. Clin Psychol Rev 57:141-163. https://doi.org/ 10.1016/j.cpr.2017.09.002

Vehmeijer FOL, Guxens M, Duijts L, Marroun HE (2019) Maternal psychological distress during pregnancy and childhood health outcomes: a narrative review. J Dev Orig Health Dis 10:274285. https://doi.org/10.1017/S2040174418000557
Wilson H, Donachie AL (2018) Evaluating the effectiveness of a dialectical behaviour therapy (DBT) informed programme in a community perinatal team. Behav Cogn Psychother 46:541553. https://doi.org/10.1017/S1352465817000790

Publisher's note Springer Nature remains neutral with regard to jurisdictional claims in published maps and institutional affiliations. 